\title{
AGUA PARA TODOS: EVALUACIÓN DE EFECTOS EN UN PROYECTO DE INNOVACIÓN SOCIAL CON COMUNIDADES INDÍGENAS*
}

\author{
WATER FOR ALL: IMPACT EVALUATION IN A SOCIAL \\ INNOVATION PROJECT WITH INDIGENOUS COMMUNITIES \\ ÁGUA PARA TODOS: AVALIAÇÃO DOS IMPACTOS \\ NUM PROJECTO DE INOVAÇÃO SOCIAL COM AS \\ COMUNIDADES INDÍGENAS
}

L'EAU POUR TOUS: EVALUATION DE L'IMPACT D'UN PROJET D'INNOVATION SOCIALE AVEC LES COMMUNAUTES INDIGENES

\section{JULIÁN DAVID SALAZAR SOLARTE'}

Fecha de recepción: 01 de marzo de 2019

Fecha de aprobación: 11 de junio de 2020

\section{RESUMEN}

La propuesta presenta la evaluación de impacto socioeconómico y ambiental de un proyecto de innovación social denominado Agua para Todos, en los resguardos indígenas del municipio de Toribío, Cauca, Colombia; proyecto mediante el cual se logró la articulación entre la empresa privada, los entes territoriales de naturaleza pública, la academia y comunidades indígenas. Metodológicamente, la investigación se abordó desde una perspectiva de enfoque cualitativo, con el propósito de caracterizar a la comunidad indígena nasa del antedicho municipio y, consecuentemente, con la evaluación de impactos derivada de la ejecución del proyecto Agua para Todos. Como herramientas metodológicas se emplearon: aplicación de

* El presente artículo es el resultado del proyecto de investigación "Evaluación de la gestión ambiental y económica en el resguardo indígena de Toribío en el marco del proyecto Agua para Todos".

Administrador de empresas, Universidad Cooperativa de Colombia, campus Popayán; beneficiario, convocatoria 06-2016, Jóvenes Investigadores; Universidad Cooperativa de Colombia, campus Popayán. juliansalazarsolarte1991@hotmail. com - ORCID 0000-0003-4618-2068.

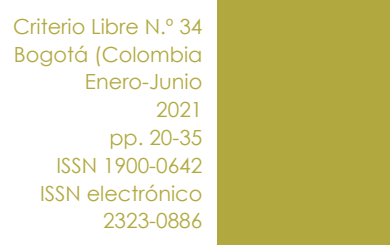




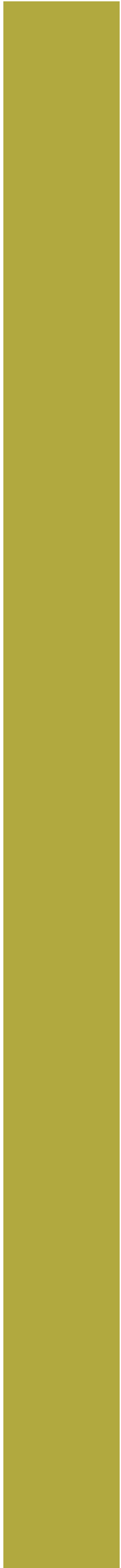

entrevistas a los directivos de Corpopalo, grupos de discusión con la comunidad beneficiaria del proyecto. Como métodos de análisis de la información se utilizaron el análisis de contenido y la categorización de variables, mediante la cual se evaluaron un grupo de categorías macro tendientes a una evaluación más eficaz: generación de ingresos, generación de empleo, calidad de vida, relacionamiento social, reorganización de fincas, respeto cultural, recuperación de fuentes hídricas, sostenimiento ambiental, mejoramiento de terrenos, sustitución de ganadería, apropiación de conciencia ambiental.

Entre los hallazgos más preponderantes están, a nivel general, el mejoramiento de las condiciones de vida de los pobladores receptores del proyecto, donde se destacan: el incremento de la capacidad productiva de las fincas, incremento de ingresos económicos derivados de las actividades agropecuarias, aumento de prácticas amigables con el medio ambiente. A su vez, una mejoría creciente de las condiciones ambientales de la zona, primordialmente de la cuenca del río Palo, entre otras.

Finalmente, se propone una serie de conclusiones entre las cuales se destaca que la innovación social marca el desarrollo de nuevas prácticas tendientes a la generación de crecimiento social, el cual, de cierto modo, se aparta de los modelos de innovación tradicionales. En consecuencia, Agua para Todos marca un derrotero para futuras y eventuales articulaciones público-privadas en sinergia con otros actores sociales, los cuales, bajo directrices de trabajo organizado, logran beneficios sustanciales para las comunidades, réditos materializados de manera directa en las condiciones de vida de sus involucrados, ejecutados en el marco de prácticas que propendan por la sostenibilidad ambiental de los territorios.

ABSTRACT

The proposal presents the evaluation of the socio-economic and environmental impact of a Social Innovation project called Water for all, in the indigenous reserves of the municipality of Toribío, Cauca, Colombia; project through which articulation was achieved between private companies, territorial entities of a public nature, academia and indigenous communities. Methodologically, the research was approached from a qualitative approach perspective, with the purpose of characterizing the Nasa indigenous community of the aforementioned municipality and, consequently, with the evaluation of impacts derived from the execution of the Water for All project. As methodological tools were used: application

PALABRAS CLAVE:

evaluación de impacto; impacto ambiental; impacto social; innovación social. 
of interviews to the directors of Corpopalo, discussion groups with the beneficiary community of the project. As for the information analysis methods, content analysis and the categorization of variables were used, through which a group of macro categories were evaluated tending to a more effective evaluation, to mention: income generation, employment generation, quality of life, social relations, reorganization of farms, cultural respect, recovery of water sources, environmental sustainability, land improvement, substitution of livestock, appropriation of environmental awareness.

Among the most preponderant findings are, at a general level, the improvement in the living conditions of the population receiving the project, where the following stand out: the increase in the productive capacity of the farms, increase in economic income derived from agricultural activities, increase of friendly practices with the environment. At the same time, a growing improvement in environmental conditions in the area, primarily in the Palo river basin, among others.

Finally, a series of conclusions are proposed, among which it is highlighted that social innovation marks the development of new practices aimed at generating social growth, which, in a way, departs from traditional innovation models. Consequently, Water for all sets a course for future and eventual public-private articulations in synergy with other social actors, which, under organized work guidelines, achieve substantial benefits for the communities, returns materialized directly in the living conditions of its stakeholders, executed within the framework of practices that promote the environmental sustainability of the territories.

Keywords: environmental impact; impact evaluation; social innovation; social impact.

JEL clasification: Q32

RESUMO

A proposta do artigo expõe a avaliação do impacto socioeconómico e ambiental de um projecto de inovação social denominado Água para Todos, nas reservas indígenas do município de Toribío, Cauca, Colômbia. Através deste projecto foi possível articular a empresa privada, as entidades territoriais de natureza pública, a academia e as comunidades indígenas. A nível metodológico, a investigação foi abordada de uma perspectiva qualitativa, a fim de caracterizar a comunidade indígena Nasa deste município e, consequentemente, com a avaliação dos impactos derivados da implementação do projecto Água para Todos. Foram utilizados os seguintes instrumentos metodológicos: aplicação de entrevistas aos directores da Corpopalo, grupos de discussão com a comunidade beneficiária do projecto. Como métodos de análise da informação foram utilizados a análise do conteúdo e a categorização das variáveis, através da qual um grupo de macro categorias foi avaliado tendendo a uma avaliação mais eficaz: geração de rendimento, geração de emprego, qualidade de vida, relação social, reorganização de explorações agrícolas, respeito cultural, recuperação de fontes de água, sustentabilidade ambiental, melhoria da terra, substituição de gado, apropriação da consciência ambiental. 


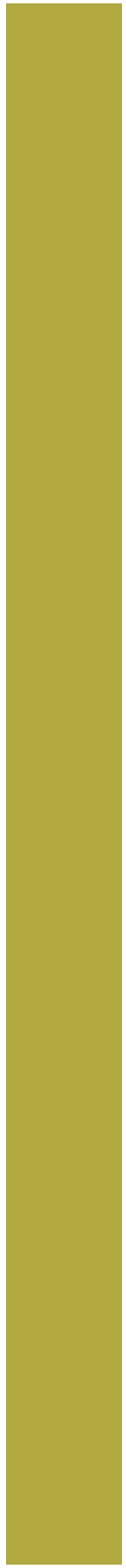

Em geral, entre os resultados mais notáveis encontram-se a melhoria das condições de vida dos beneficiários do projecto, onde se destacam: o aumento da capacidade produtiva das explorações agrícolas, o aumento do rendimento económico derivado das actividades agrícolas, o aumento das práticas amigas do ambiente. E ao mesmo tempo, uma melhoria crescente das condições ambientais da área, principalmente da bacia do rio Palo, entre outras.

Finalmente, é proposta uma série de conclusões, entre as quais se destaca que a inovação social marca o desenvolvimento de novas práticas destinadas a gerar crescimento social, que, de certa forma, se afasta dos modelos tradicionais de inovação. Por esta razão, Água para Todos marca um caminho para futuras e eventuais articulações público-privadas em sinergia com outros actores sociais, que, sob orientações de trabalho organizadas, obtêm benefícios substanciais para as comunidades, com retornos materializados directamente nas condições de vida dos envolvidos, executados no quadro de práticas que favorecem a sustentabilidade ambiental dos territórios.

Palavras-chave: avaliação de impacto; impacto ambiental; impacto social; inovação social.

Classificação JEL: Q32

La proposition de l'article expose l'évaluation de l'impact socioéconomique et environnemental d'un projet d'innovation sociale appelé Eau pour tous, dans les réserves indigènes de la municipalité de Toribío, Cauca, Colombie. A travers ce projet il a été possible d'articuler l'entreprise privée, les entités territoriales de nature publique, l'académie et les communautés indigènes. Au niveau méthodologique, la recherche a été abordée dans une perspective qualitative, afin de caractériser la communauté indigène Nasa de cette municipalité et, par conséquent, avec l'évaluation des impacts dérivés de la mise en œuvre du projet Eau pour tous. Les outils méthodologiques suivants ont été utilisés: application des entretiens aux directeurs de Corpopalo, groupes de discussion avec la communauté bénéficiaire du projet. Comme méthodes d'analyse de l'information, on a utilisé l'analyse du contenu et la catégorisation des variables, à travers lesquelles on a évalué un groupe de macro-catégories tendant à une évaluation plus efficace: génération de revenus, génération d'emploi, qualité de vie, relation sociale, réorganisation des exploitations, respect culturel, récupération des sources d'eau, durabilité environnementale, amélioration des terres, remplacement du bétail, appropriation de la conscience environnementale.

D'une manière générale, l'amélioration des conditions de vie des bénéficiaires du projet est l'un des résultats les plus marquants, avec notamment : l'augmentation de la capacité de production des exploitations, l'augmentation des revenus économiques issus des activités agricoles, l'augmentation des pratiques respectueuses de l'environnement. Et en même temps, une amélioration croissante des conditions environnementales de la région, principalement du bassin du fleuve Palo, entre autres. 
Enfin, une série de conclusions sont proposées, parmi lesquelles il est souligné que l'innovation sociale marque le développement de nouvelles pratiques visant à générer une croissance sociale, ce qui, d'une certaine manière, s'écarte des modèles d'innovation traditionnels. Pour cette raison, l'Eau pour tous marque une voie pour les futures et éventuelles articulations public-privé en synergie avec d'autres acteurs sociaux, qui, dans le cadre d'un travail organisé, permettent d'obtenir des bénéfices substantiels pour les communautés, avec des retours directs matérialisés dans les conditions de vie des personnes impliquées, exécutés dans le cadre de pratiques qui favorisent la durabilité environnementale des territoires.

Mots clés: évaluation d'impact; impact environnemental; impact social; innovation sociale.

\section{Classification JEL: Q32}

\section{INTRODUCCIÓN}

Los nuevos procesos de innovación y de emprendimiento social pueden plantearse como alternativas a la solución de problemáticas sociales, económicas y medioambientales de las comunidades. Dichos procesos han tomado una importante relevancia a partir de las últimas décadas del siglo pasado y se han convertido en tema central para la agenda de muchos estamentos gubernamentales y privados a nivel mundial. La innovación social, según Philis, Deiglmeier y Miller (2008), es una solución novedosa a un problema social más eficaz, eficiente, sostenible o justa.

Es así como en el año 2010, por iniciativa de las comunidades indígenas del municipio de Toribío, Cauca, Colombia, nace el proyecto que a la postre se denominaría Agua para Todos; un proceso de gestión socio-económica y ambiental que propendía por la preservación de los espacios de vida y de la cuenca del río Palo' en esa región, mediante la implementación de prácticas agrícolas y pecuarias, las cuales les permitieran tener un mejoramiento sustancial en sus condiciones de vida. Dicho proyecto plantó una semilla de relacionamiento directo, con el cual se buscó una sinergia entre diferentes actores, como lo fueron la comunidad, la empresa privada, la academia y las instituciones públicas, dinamizando la interacción entre las mismas con la búsqueda de un fin común.

El municipio de Toribío se encuentra sobre el costado occidental de la cordillera central, al nororiente del departamento del Cauca, con una altura máxima de 4.150 m.s.n.m. en el páramo de Santo Domingo. Se encuentra conformado por tres resguardos indígenas ${ }^{2}$ (Tacueyó, Toribío y San Francisco). Tiene una población de más de 30.000 personas, de las que aproximadamente $94,5 \%$ residen en zonas rurales.

1 Río Palo: importante afluente hidrográfico de la zona, con un área aproximada de 152.000 hectáreas y un recorrido de $92 \mathrm{~km}$, perteneciente a la gran cuenca del Río Cauca (segundo en importancia en Colombia). Dentro de esta subcuenca existen ecosistemas de gran importancia para la zona de influencia.

2 Los resguardos indígenas son propiedad colectiva de las comunidades indígenas a favor de las cuales se constituyen y conforme a los artículos 63 y 329 de la Constitución Política, tienen el carácter de inalienables, imprescriptibles e inembargables. Los resguardos indígenas son una institución legal y sociopolítica de carácter especial, conformada por una o más comunidades indígenas, que con un título de propiedad colectiva que goza de las garantías de la propiedad privada, poseen su territorio y se rigen para el manejo de este y su vida interna por una organización autónoma amparada por el fuero indígena y su sistema normativo propio. Decreto 2164 de 1995, artículo 21. 
Principalmente su economía se basa en la agricultura, siendo el café, plátano, maíz, entre otros, los productos más destacados dentro de su economía productiva. Sumado a esto, la ganadería reviste un renglón importante dentro de su componente financiero. Por otra parte, de acuerdo con datos suministrados por la Alcaldía de Toribío (2019), el municipio cuenta con aproximadamente 50.000 hectáreas de extensión, de las cuales cerca de $42 \%$ son consideradas zonas de reserva; dicha etnia considera las fuentes hídricas como espacios de vida y es por lo que trabajan en su protección continua.

En el año 1980 se crea el Proyecto Nasa, con el objetivo de generar vínculos de unión entre los cabildos. La composición nuclear de los resguardos concentra su plan de vida en cuatro ámbitos.

1. Familia: dentro de este núcleo se desarrollan los asuntos de salud, educación y familia; en el primero de ellos destaca el esfuerzo que se realiza como comunidad para recuperar los valores y principios ancestrales; en el marco de estos procesos, la comunidad cuenta con un sistema de educación propio que busca principalmente la recuperación de la lengua e identidad, característicos de su cultura. En el marco de la salud, recuperar el empleo de las plantas medicinales más tradicionales en su historia y, en el marco familiar, la restauración de la familia nuclear.

2. Comunidad: revitalización de prácticas ancestrales, en concordancia con las políticas y lineamientos del plan de vida.

3. Territorio: corresponde al ejercicio de lo productivo, ambiental, empresarial; busca recuperar la autonomía alimentaria que en décadas pasadas existía dentro de la comunidad, en las cuales las fincas y familias eran autosuficientes en su producción y subsistencia.

4. Gobierno: destinado a la dinamización de la comunidad mediante la implantación de políticas.

Culturalmente, los pobladores de la comunidad nasa realizan a lo largo del año una serie de rituales que están consagrados dentro de sus leyes de origen y naturales. Los definen como espacios y tiempos para equilibrar, armonizar y cumplir derechos de la naturaleza. (Proyecto Nasa, 2019).

Dentro de la identidad de la comunidad nasa se encuentra el plan de vida concordante con su cosmovisión. Su principal objetivo se encuentra orientado a la autodeterminación, siendo esta la orientadora de una reorganización fortalecida encaminada a la recuperación de los territorios considerados ancestrales, de propiedad colectiva. De igual manera, en busca del fortalecimiento del ejercicio de la autoridad tradicional, basada en procesos de carácter participativo. El plan de vida nasa se encuentra revitalizado porque se sitúa bajo los preceptos orientados por los espíritus.

El sentir nasa parte de la base de una serie de principios que les da identidad como pueblo; la espiritualidad nace de la energía que proporciona la madre tierra y los espíritus guardianes para que de tal manera se pueda vivir y gobernar con fuerza, determinación y sensibilidad. Por esta razón, se debe armonizar a todos los pobladores desde sus prácticas culturales. El ser gobierno otorga el poder de cuidar, defender el territorio y orientar a la comunidad a partir de los mandatos espirituales y naturales. Finalmente, el ser territorial marca una simbología que le da identidad a cada uno de los elementos que emplean en su cotidianidad:

Hay diversos tejidos nasa, la mochila, que tiene una función que es para guardar semillas y se relaciona con el útero de la mujer, las mochilas de compromiso como la cuetandera, el anaco, faja para cubrir, proteger los genitales de la mujer, la ruana y la cobija para proporcionar calor al cuerpo de la familia, el taw "chumbe nasa" que es para mantener la memoria y el pensamiento ancestral y recrear el pensamiento de la vida nasa, la vivienda nasa, tejido que abriga, protege la familia y garantiza la continuidad de las generaciones como pueblo nasa. (Proyecto Nasa, 2019). 


\section{- APROXIMACIÓN TEÓRICA}

Innovación, en un sentido amplio y general, según Boni, Belda-Miquel, y Pellicer-Sifres (2018), que a su vez retoman a Freeman (1991), la definen como la capacidad que tienen los seres humanos para explotar una idea o un nuevo método que le permita alcanzar un efecto deseado, bien sea material o social, dentro de lo cual se puede abarcar desarrollos tecnológicos, procesos, organizaciones y nuevos servicios. De igual manera, la Universidad Católica de Chile (2012) asocia este concepto a la creación de algo nuevo, llámese producto o proceso.

Con relación a la innovación social, Mulgan \& Sanders (2007) la destacan como ciertas actividades y servicios innovadores motivados por la satisfacción de necesidades sociales que permitan con el tiempo alcanzar un modelo de escalabilidad y replicabilidad. En ese mismo sentido, Westley (2009) la refiere como un proceso complejo dentro del cual se introducen productos, procesos o programas que modifican connaturalmente las rutinas básicas, recursos y el flujo de autoridad o de creencias respecto del sistema social donde surgen, resaltando impersonalmente que las innovaciones exitosas de este tipo perduran en el tiempo y generan un impacto importante.

Por otra parte, Sánchez Álvarez (2012), quien retoma a Howaldt y Schwarz (2010), establece que la innovación social es una nueva combinación o configuración de prácticas sociales en ciertas áreas de acción o en contextos sociales definidos por los actores o una constelación de ellos con el objetivo de satisfacer o responder a necesidades o problemas. Así mismo, Quijano y otros (2017), retomando a Bueno y Pizarro (2011), establecen la innovación social como el proceso o resultado de introducir un cambio para la mejora o el progreso de un conjunto de individuos que interactúan entre sí para conformar una comunidad.

Dicha información se puede condensar bajo un supuesto que plantea la introducción de una novedad encaminada a la resolución de los problemas sociales de la comunidad.

Prandi y Cano (2015) determinan la relevancia de dos elementos dentro de la innovación social, el primero de ellos lo estipulan como lo novedoso o efectivo de la idea en el momento de implementarse, y en segundo lugar, la primacía del bien común al ser esa idea una condensadora de beneficios que permiten la resolución de un problema social. Echeverría (2008) fue más allá de cualquier tipo de concepto generalizado y la asocia directamente a valores sociales, como lo son el bienestar, la calidad de vida, la inclusión social, la solidaridad, la participación ciudadana, la calidad medioambiental, la atención sanitaria, la eficiencia de los servicios públicos o el nivel educativo de una sociedad; ratifica dicha afirmación al mencionar que una innovación social es relevante en la medida en que se oriente a valores sociales, no solo a la productividad ni a los elementos inherentes a la temática empresarial. Además, relaciona directamente la innovación social con calidad de vida, la cual aporta ventajas competitivas entre sociedades. Del mismo modo, hace un aporte importante al considerar que un proceso de innovación social exitoso debe ser medido, a priori, en función del grado de aceptación social de dichas iniciativas, ya que de no lograr la receptividad deseada se dificulta la continuidad del proceso, todo esto bajo el supuesto de que debe haber un trabajo mancomunado y cooperativo entre los entes que desean intervenir en determinada comunidad y sus beneficiarios directos.

Como se pudo evidenciar, las aproximaciones teóricas de los diferentes autores difieren de cierta manera en la composición de los elementos constitutivos; cabe mencionar que la innovación social tiene un solo fin, y es que mediante su implementación se busca la solución de problemas sociales, considerando de tal modo el mejoramiento de las condiciones de vida de los usuarios y encauzando una sinergia entre la comunidad y los entes dispuestos para ejecutar tales proyectos, políticas, procesos. etc. Finalmente, Buckland y Murillo (2014) 
determinaron un conjunto de cinco variables para entender la innovación social que resulta relevante mencionar: la primera es el impacto social, seguido de la sostenibilidad económica, el tipo de innovación, colaboración intersectorial y la escalabilidad y replicabilidad.

Cohen y Martínez (2002) establecieron que el impacto de un proyecto o programa social es la magnitud cuantitativa del cambio en el problema de la población, en la cual se mide la comparación entre datos e información de una línea base a una situación posterior que puede ser denominada línea de comparación. El impacto de un proyecto, programa o proceso se da cuando se asocian dichos resultados al problema inicial que se planteó como punto de partida. Franks (2012), quien a su vez retoma a Vanclay y otros (2015), describe el impacto social como algo que se experimenta 0 se siente por un individuo, grupo social o unidad económica; los impactos sociales son el efecto de una acción -o quizá también de una omisión- con resultados que pueden ser tanto positivos como negativos. Teniendo en cuenta que se basa en criterios subjetivos, se debe entender el impacto como algo que abarca los resultados de una acción independiente de si son benéficos o no para los involucrados (Franks, 2011).

Canter (1997) establece que el impacto ambiental se presenta cuando una acción y consecuencia de un programa - proyecto produce una alteración, favorable o desfavorable, en el medio o en alguno de los elementos que lo componen. Fernández (1993) destaca que el impacto de un proyecto sobre el medio ambiente es la diferencia entre la situación del medio ambiente futuro modificado, tal como se manifestaría como consecuencia de la realización del proyecto y, a su vez, de la evolución que habría tenido ese mismo entorno sin la intervención propuesta.

De igual manera, Garmendia y otros (2005) conceptualizan el impacto ambiental como la alteración de la calidad del medio ambiente producida por una actividad humana; entre tanto, el suponer hablar de impacto ambiental se debe encauzar la línea de investigación hacia las acciones humanas, entendiendo que dichos efectos o impactos distan de los agentes externos derivados de la propia naturaleza, y su propósito esencial se centra en la evaluación de una obra, actividad o programa. De tal manera, se puede inferir que la Evaluación de Impacto Ambiental (EIA) es un procedimiento analítico encaminado a la elaboración de un juicio de carácter objetivo que permite identificar los impactos o consecuencias que se derivan de una intervención o actividad.

Nuevamente, Cante (1997) reafirma que la Evaluación de Impacto Ambiental (EIA) es un procedimiento jurídico/administrativo que tiene como propósito la identificación, predicción einterpretación de losimpactos ambientales que un proyecto o actividad produciría en caso de ser ejecutado, al igual que la prevención, corrección y su valoración. Por su parte, Garmendia y otros (2005) recalan en la Evaluación de Impacto Ambiental (EIA) y se refieren a ella como una valoración de los impactos que se generan sobre el medio ambiente a través de un determinado proyecto; el énfasis de este tipo de estudios debe ser netamente subjetivo, ya que su referencia principal es la calidad ambiental.

Resulta necesario mencionar la existencia de una alternativa en lo referente al momento en que se pretende evaluar los impactos de un proyecto o programa que no se anticiparon. Navarro (2005) plantea que, para desarrollar el trabajo de esta manera, no se requiere la predefinición de variables de impacto ni tampoco se asignan controles para aislar la incidencia de factores externos. Tal como lo afirma Stufflebeam (1999) al mencionar que la importancia de la evaluación que no anticipa los efectos radica en que dichos resultados pueden, en determinado momento, llegar a ser más relevantes que en aquellos casos en que sí fueron previstos.

La estrategia de evaluar bajo esta modalidad consiste en la utilización de métodos cualitativos ya que no se emplea el pre-establecimiento de variables. Schutt 
(2001) determinó que el uso de un método cualitativo emplea un pensamiento que denomina ideográfico, dentro del cual se identifican una sucesión de eventos que ocurren en diferentes momentos y que conducen a la generación de impactos. Navarro (2005) hace un aporte importante al precisar que este método emplea un "enfoque inductivo de investigación" dentro de la cual se obtienen conceptos finales y generales a partir de premisas de carácter particular; dentro de este enfoque no se identifican a priori los posibles efectos, y por tanto, no se formula ningún tipo de hipótesis.

\section{2. metodología y ANÁLISIS}

La investigación se efectuó bajo un enfoque de carácter cualitativo (Hernández (2014), con el propósito de identificar los impactos más relevantes arrojados por el proyecto Agua para Todos en los resguardos indígenas de Toribío, Cauca, Colombia, basados en las experiencias y percepciones de sus actores. En desarrollo del proyecto de investigación se planteó un objetivo macro, denominado Evaluar la gestión ambiental y económica en los resguardos indígenas del municipio de Toribío en el marco del proyecto Agua para Todos.

Como objetivos específicos se plantearon, en primera medida: caracterizar la zona referente a aspectos económicos, sociales y ambientales; como segundo objetivo, identificar las prácticas implementadas por Corpopalo a través del proyecto Agua para Todos en los cabildos de Toribío dentro de los últimos cinco años: un tercer objetivo, denominado Develar la concepción de la comunidad referente a las prácticas implementadas a través del proyecto Agua para Todos.

El componente metodológico descrito tiende a evaluar los impactos socioambientales de una manera cualitativa, teniendo en cuenta que, mediante el rastreo documental y el abordaje a las directivas encargadas de la ejecución, se pudo precisar que el proyecto no develaba indicadores de impacto, ya que su ejecución no partió de una línea de base documentada, y por tal motivo no se puede develar una exposición objetiva por no existir datos de referencia, la investigación se encaminó a la recopilación de información cualitativa, donde se tuvo en cuenta a sus actores principales: la comunidad y los entes ejecutores, ${ }^{3}$ en este caso Corpopalo. ${ }^{4}$

En la primera fase del desarrollo metodológico se asume una revisión bibliográfica de la temática propuesta, en la cual, además del aparte teórico fue necesario acudir a fuentes que permitieran hacer un rastreo de la comunidad, caracterizarla en sus costumbres, cultura, tradiciones; labor en la que se involucró una recopilación documental y a su vez fue alimentada con el posterior trabajo de campo. Seguido de ello fue necesario movilizar los esfuerzos hacia el conocimiento pleno del proyecto Agua para Todos, partiendo de la base de la identificación de los procesos ejecutados desde sus inicios, sus avances y las actividades ejecutadas a través de las mismas; en este abordaje, la investigación se orientó a la recopilación documental de informes del proyecto $y$, a su vez, en el momento de ejecutar el trabajo de campo se compilaron más datos de gran relevancia para la investigación.

Posteriormente se elaboraron dos instrumentos de investigación, el primero de ellos una entrevista semiestructurada aplicable a los directivos de la Corporación, constituida por dos directrices específicas, la primera de ellas propuesta con el fin de entender los objetivos de intervención del proyecto, las fases en que se ha desarrollado $y$, en segundo lugar, la determinación de impactos socio-ambientales. Para el examen crítico y objetivo de la información recopilada se procede al empleo del análisis de contenido; al respecto, Andreú (2012) la define como una técnica encaminada a la interpretación de textos, teniendo en cuenta estos últimos en su

\footnotetext{
3 Agua para Todos es una iniciativa público-privada junto con la academia.

4 Corporación para el manejo integral y recuperación de la cuenca del río Palo. Entidad encargada de la gestión y ejecución del proyecto Agua para Todos.
} 
mayor entendimiento al referirlos como aquellos en los que se incluye lo que no necesariamente se encuentra escrito, donde se pueden incluir transcripción de entrevistas, discursos, entre otros. En ese sentido, Holsti (1969) determina el análisis de contenido como una técnica de investigación tendiente a formular inferencias mediante la identificación sistemática y objetiva de ciertas características dentro de un texto. Bajo este enfoque, el aporte realizado por Holsti resulta imprescindible al esbozar el análisis de contenido como un mecanismo idóneo para determinar precisiones con información cualitativa, al añadirle un importante elemento: la inferencia, con el cual se busca determinar los efectos del proyecto basados en un ejercicio de retrospección comparativa inclinada hacia la detección de esas variables.

En la segunda fase de desarrollo metodológico se implementó un grupo de discusión con la comunidad beneficiaria del proyecto Agua para Todos; dicho trabajo se efectuó con 22 comuneros de diferentes fincas, encaminado a la determinación de dos aspectos: el primero de ellos tendiente a la caracterización de la comunidad y el segundo a la identificación de los impactos arrojados por el proyecto en los últimos años. En ese sentido, posteriormente a la recopilación de la información contenida en los dos instrumentos se procede al análisis efectuado bajo las siguientes categorías: generación de ingresos, generación de empleo, calidad de vida, relacionamiento social, reorganización de fincas, respeto cultural, recuperación de fuentes hídricas, sostenimiento ambiental, mejoramiento de terrenos, sustitución de ganadería, apropiación de conciencia ambiental.

La ejecución del proyecto Agua para Todos trajo consigo la implementación de prácticas amigables con el medio ambiente, tanto en agricultura como en ganadería, todo esto en concordancia con el plan de vida "Proyecto Nasa", en busca de la protección de los espacios de vida sin menoscabo de las tradiciones culturales. Es de destacar que el respeto a las tradiciones marcó un punto de convergencia entre las relaciones de la comunidad y los entes intervinientes del proyecto, evidenciándose cada actividad que se pretendía realizar con relación a los preceptos indígenas. A continuación se presentan los impactos más representativos del proyecto, los cuales se cimientan en tres aristas principales: la primera en un ámbito social, seguido de uno medioambiental y finalmente, el componente económico.

Socialmente se ha logrado contribuir a la satisfacción de algunas necesidades humanas fundamentales de la comunidad, resaltándose las fisiológicas, de seguridad, afiliación, reconocimiento y autorrealización. Se logró implementar mejores formas de hacer las cosas, combinando sus conocimientos ancestrales con los occidentales, encontrando una relación entre ambos.

De igual manera, se logró que a través de las capacitaciones proporcionadas por Corpopalo hubiera generación de nuevos hábitos agropecuarios y la apropiación del conocimiento en aras de la ejecución en las labores de las fincas. Se logra evidenciar de igual manera la unión entre la comunidad, siendo uno de los factores más importantes la colaboración entre los comuneros, en consideración a que, previa intervención de la zona, los pobladores realizaban sus prácticas de una manera autónoma e independiente sin que las labores más destacadas fueran conocidas por los demás, aspecto que se logró cambiar con la implementación de Agua para Todos, con la consolidación del trabajo en equipo dentro de la comunidad. La apropiación de los procesos emprendidos se realiza con entusiasmo, y es de esta manera como se ha logrado la participación de madres, niños(as) y jóvenes, aspecto que primordialmente deja sentadas las bases para la sostenibilidad del proyecto y de prácticas que se desee efectuar en el futuro.

Se puede mencionar que la calidad de vida de los beneficiarios del proyecto ha tenido una mejoría evidente, en razón a que, mediante la reorganización de las fincas, independientemente de cuál sea su naturaleza (comunitaria o familiar) se ha logrado que las jornadas de trabajo 


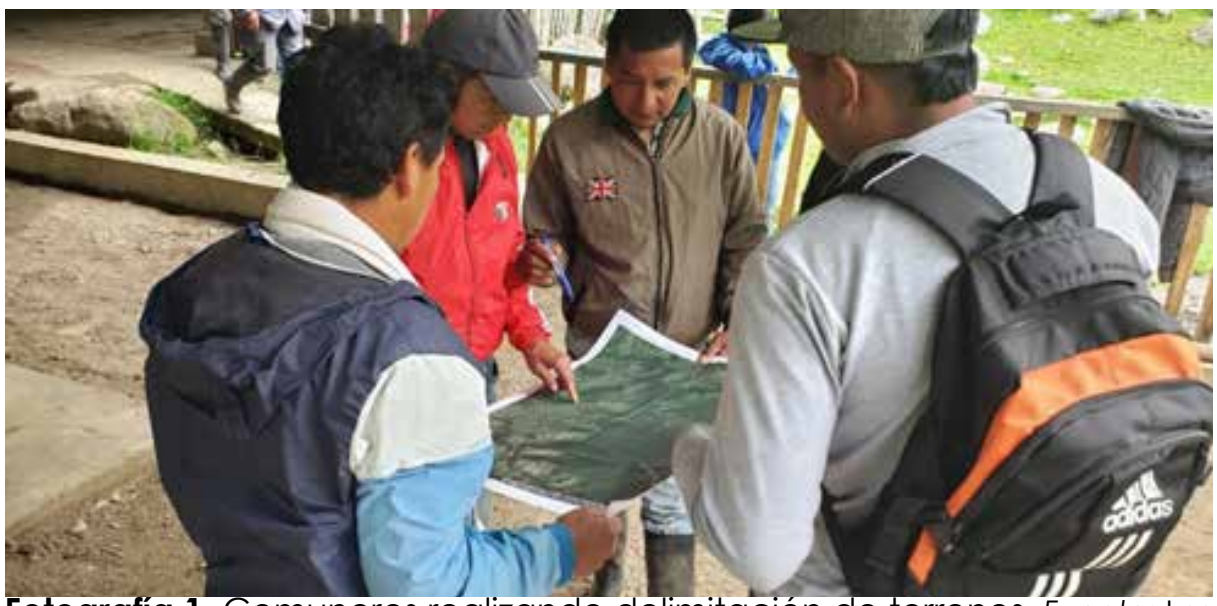

Fotografia 1. Comuneros realizando delimitación de terrenos. Fuente: Los autores.

no sean tan dispendiosas, en el sentido de tener, por ejemplo, el ganado organizado en pequeñas extensiones, lo que da mayor posibilidad de realizar las actividades de ordeño en menor tiempo. Todo esto con motivo de que, previo a la ejecución del proyecto Agua para Todos, la labor de ubicación del ganado resultaba dispendiosa, toda vez que los comuneros debían recorrer grandes extensiones de tierra para poder efectuar esta actividad. Lo precedentemente retratado va de la mano con las apreciaciones hechas por Franks (2012), al plasmar en su literatura que la base de la evaluación social de un proyecto radica en la experiencia de los individuos, aquellos que pueden aportar de sus vivencias propias la base de la valoración de algo.

Finalmente, el empoderamiento de la comunidad ha sido un punto clave, la restauración de sus valores ancestrales, alineado con la preservación de los espacios de vida ha permitido a los pobladores reconocer que mediante el proyecto se ha logrado apropiar considerablemente al individuo dentro de su entorno social y natural.

En la dimensión ambiental, cabe mencionar que las familias beneficiarias del proyecto cuentan con un sentido de conciencia ambiental alto. Se ha contribuido sustancialmente en el ordenamiento del río Palo al implementar herramientas como las cartográficas, lo cual ha permitido una mejor planeación y ordenamiento del territorio (Fotografías 1 y 2).
Con la implementación del proyecto se han logrado demarcar extensas áreas de protección, conservación y regeneración natural, aislando al ganado y consiguiendo evitar la tediosa y perjudicial (en términos ambientales) ganadería extensiva. A través de Agua para Todos se ha podido aislar una considerable cantidad de nacimientos de agua, evitando el pisoteo del ganado $y$, consecuentemente, la protección de los recursos hídricos, evidenciándose una regeneración que, según la concepción de la comunidad, es significativa. La Fotografía 3 denota la importancia del mejoramiento de la calidad de vida de los pobladores con relación a la sustitución de la ganadería extensiva por una intensiva en la cual se determina una mejoría en las condiciones laborales de los comuneros con relación a la disminución de tediosas jornadas de trabajo en las cuales anteriormente, por lo extenso de sus territorios, destinaban gran parte de su tiempo en el agrupamiento del ganado y todas sus actividades derivadas.

De igual manera, se ha propendido por evitar la tala indiscriminada de árboles y la implantación de cercas vivas con un porcentaje alto de éxito, mejorando visiblemente las condiciones de las fincas y contribuyendo a la restitución de vegetación en vez del empleo de postes de madera muerta o inclusive cemento. Con relación a esto, la comunidad ha tomado conciencia de que habitan una zona de vocación forestal, razón por 
la cual destinaron áreas de sus mismas fincas para aislarlas y permitir que el bosque se regenere naturalmente. De igual manera, la repoblación de bosques se ha hecho con especies arbóreas nativas, como lo son el encenillo, palma de cera, lechero, cedro, entre otros; variedades que permiten un buen acoplamiento con la naturaleza al ser originarias de la zona. Finalmente, al ser indagados, los pobladores dan razón de que las condiciones de la cuenca del río Palo se han modificado considerablemente, debido a que, con las prácticas responsables han logrado tener un cuerpo de agua más limpio y con un cauce más alto que cuando se inició el proyecto, argumentando de igual manera que en época de sequía el río no se seca, situación que se presentaba en años pasados. En la Fotografía 4 se puede contrastar el aislamiento y regeneración de pasturas en comparación con una zona no intervenida.
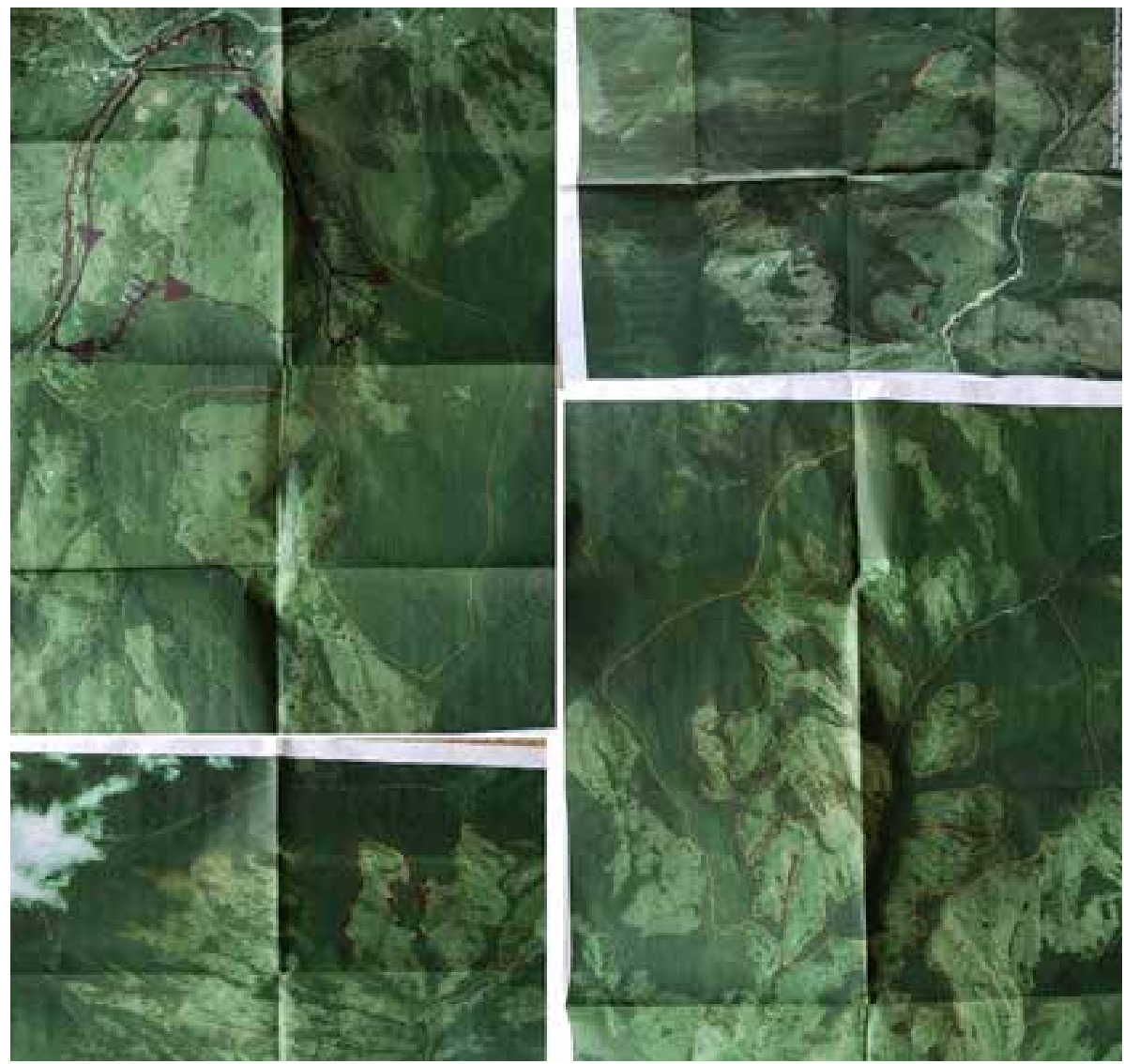

Fotografía 2. Registros cartográficos de fincas beneficiarias. Fuente: Los autores. 


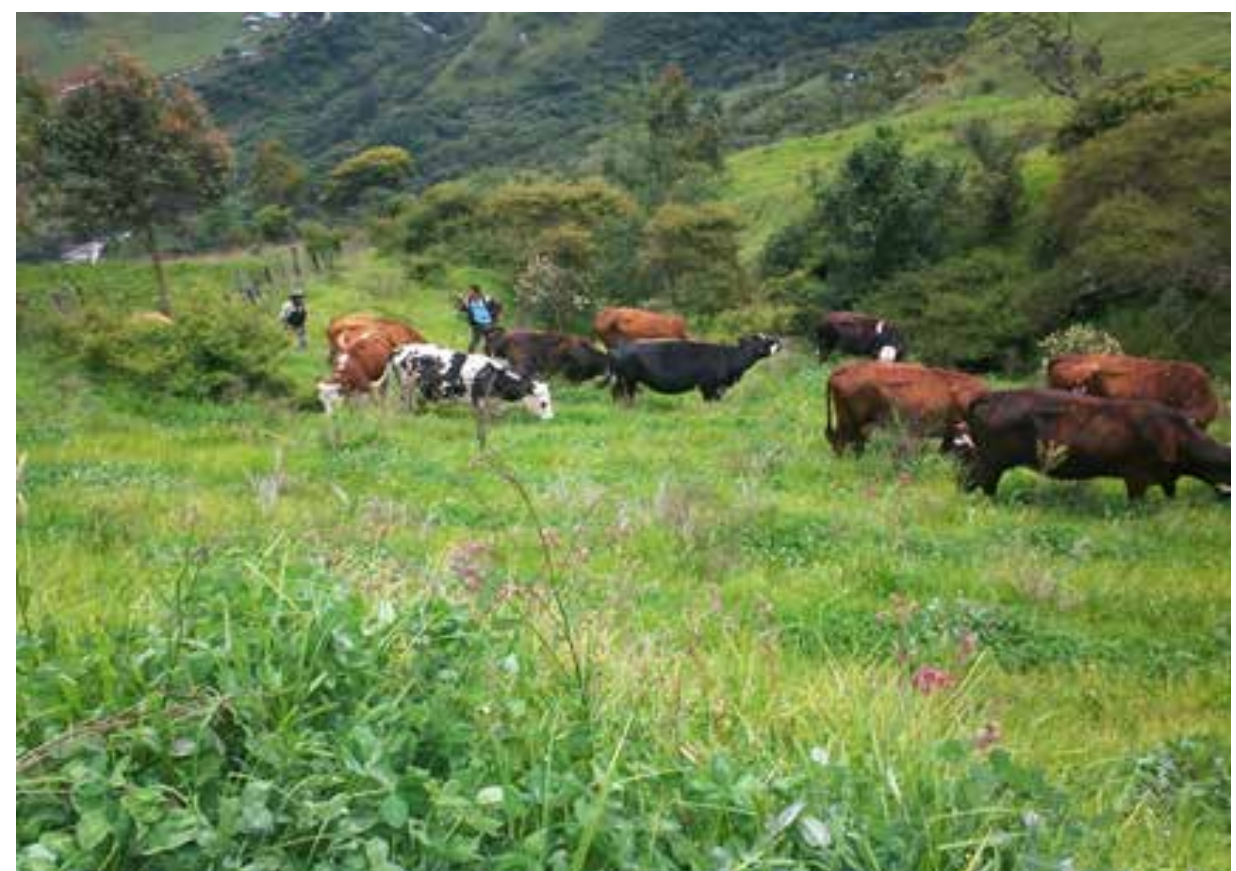

Fotografía 3. Producción ganadera, comunidad indígena nasa, resguardo de Tacueyó. Fuente: Los autores.

Algunos pobladores mencionaron que, gracias a Agua para Todos, algunas familias han logrado en poco menos de cinco años lo que no habían alcanzado en veinte años, todo esto con relación al cambio observado en la protección de los espacios de vida, mejores prácticas ganaderas, producción de alimentos y el empoderamiento de las familias.

Abiertamente se han destacado los principales impactos de carácter positivo del proyecto, vale la pena hacer destacar someramente algunos retos que en concepto de la comunidad se deben trazar en la ejecución de nuevas etapas del proyecto. Aunque la calidad de vida en lo económico ha mejorado, se considera que en las fincas más pequeñas no hay trabajo para todos, y por tanto se debe acudir a fuentes externas, ya sea en otras fincas o inclusive en la inserción de cultivos ilícitos. Esta última de constante preocupación para los comuneros, al referir que este tipo de prácticas han desplazado el trabajo tradicional por las facilidades que les brindan personas al margen de la ley en el sentido de la oferta y demanda. En consecuencia, la facilidad para comercializar este tipo de productos es mayor que en aquellos que se producen lícitamente dentro de sus fincas. Otro factor importante ha sido que el municipio cuenta con más de 49.000 hectáreas de extensión rural y, debido a esta complejidad se calcula que el proyecto ha llegado a solo $2 \%$ de toda esta zona, razón por la cual las personas piden una mayor intervención por parte de Corpopalo. De igual manera, resaltan que al ser zonas de gran extensión se dificulta el mantenimiento de las áreas, y por tanto, se presenta en alguna de ellas un deterioro inminente de lo ya ejecutado.

\section{CONCLUSIONES}

La innovación social ha marcado el desarrollo de nuevas prácticas en aras de la búsqueda y generación de un crecimiento social que se desliga, de cierto modo, de la innovación en un carácter netamente económico, saliendo del paradigma de la producción industrial y pasar a un terreno más social, en el cual se involucran las comunidades y se realizan intervenciones, con actividades tendientes al mejoramiento de la calidad de vida de las personas con la plena delimitación, satisfacción y resolución de necesidades.

Se debe velar por la difusión de este 


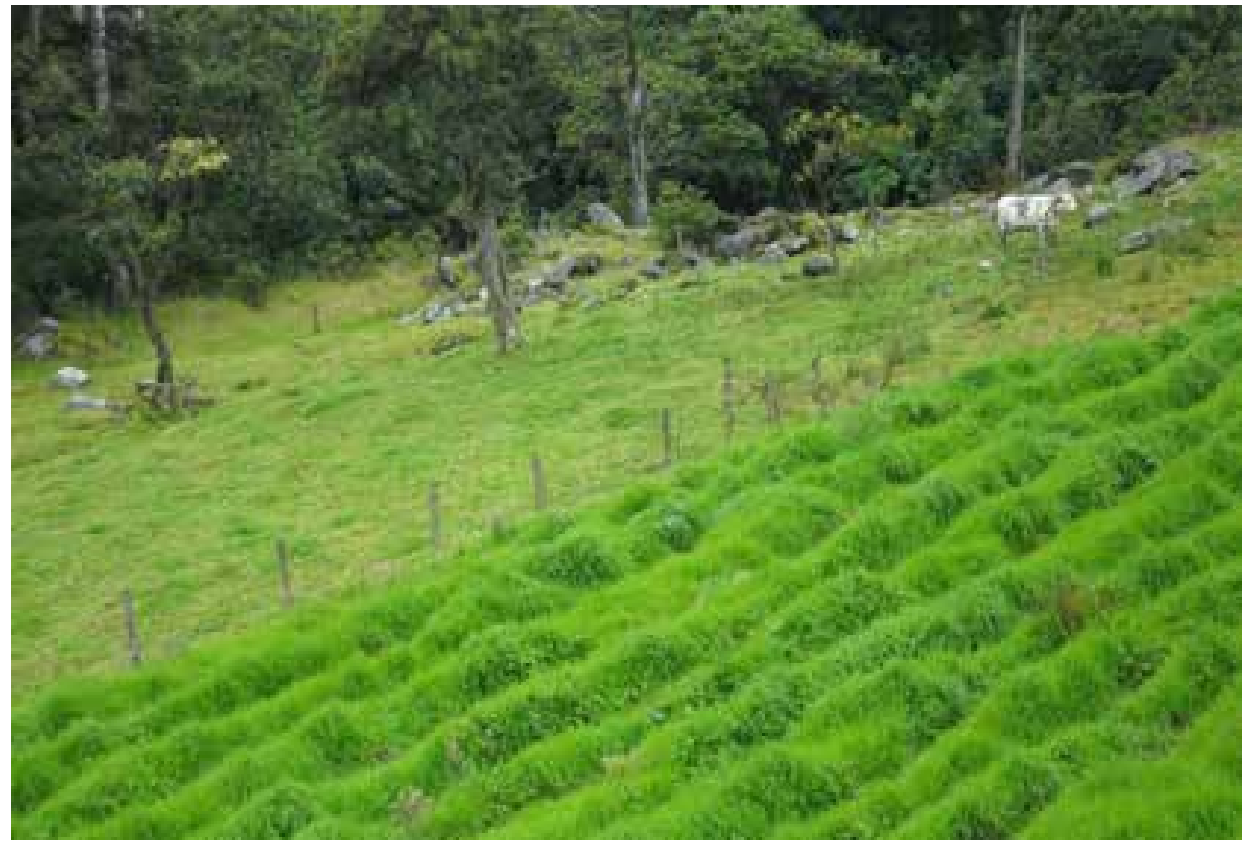

Fotografía 4. Aislamiento y regeneración de pasturas. Fuente: Los autores.

tipo de proyectos, toda vez que en la sociedad moderna adquieren un papel preponderante, ya que la innovación social cuenta con preceptos que destacan el involucramiento de los actores sociales, que buscan una sinergia representativa entre todossusintervinientes, estableciendo medidas que van de la mano con el respeto a los aspectos culturales y sociales, poniendo de presente que cualquier actividad que se busque ejecutar debe ir en consonancia con la representatividad comunitaria y no menoscabar ni reprimir de ningún modo las creencias con fines netamente comerciales.

Como se evidenció, se ha podido determinar una serie de impactos generados a través del proyecto. La dificultad radica, comolo mencionaban los directivos de Corpopalo, en la naturaleza más espontánea que planeada, ya que el intercambio de saberes ha permitido identificar las necesidades de la comunidad de una forma inmediata y de este modo ejecutar las actividades que han surgido con el paso del tiempo.

En el momento de poder realizar una evaluación de impacto totalmente eficiente se debe contar con información más objetiva, por lo que resulta pertinente quealejecutarproyectosdeestanaturaleza se propenda por determinar una línea base e identificar las variables que a la postre permitirán identificar fehacientemente los aspectos más relevantes de cualquier tipo de intervención.

Agua para Todos es muestra de la articulación entre diversos actores, destacándose que los beneficiarios, al ser una comunidad indígena se encontraban dotados de características socio-culturales que de cierto modo limitaban cualquier tipo de intervención, pero con esto se demuestra que al encontrar un punto de equilibrio donde el respeto por lo ancestral puede ir de la mano con el desarrollo, es aquí donde la comunidad logra un mejoramiento en sus condiciones de vida, de la mano con el amparo de sus dogmas espirituales y la colaboración intersectorial que propende por un bien común.

Finalmente, es importante resaltar que el proyecto Agua para Todos marca un hito de replicabilidad futura, siendo este un proyecto inédito en el cual concurre una efectiva articulación entre actores, destacándose la voluntad de una comunidad indígena en interactuar con entes que hasta hace poco tiempo eran ajenos a ellos, y 
lograr efectivamente una asociatividad encaminada a la consecución de fines basados en relaciones de respeto y reciprocidad, encauzadas a la protección del medio ambiente y la generación de mejores condiciones de vida para quienes intervienen en ella. Resultan significativos los esfuerzos articulados, aunque hay muchos aspectos por trabajar en el futuro en la diversificación de la economía local; Agua para Todos es un claro ejemplo de proyectos que abandonan de cierta manera la generación de riqueza y se encaminan a la protección de fines comunes como la apropiación social y los efectos ambientales de las actividades humanas.

Agradecimientos: A la Corporación para el manejo integral y recuperación de la cuenca del río Palo en cabeza de sus directivas, su grupo de trabajo de campo y la comunidad del resguardo indígena de Tacueyó en Toribío, Cauca, Colombia.

\section{REFERENCIAS}

Dimensión Ambiental. (s. f.). Gov.co. Recuperado 31 de agosto de 2021, de http://www.toribio-cauca.gov.co/ municipio/ecologia

Abela., J. A., \& Andaluces., I. S. F. C. (s. f.). Las técnicas de Análisis de Contenido: Una revisión actualizada. Mastor.cl. Recuperado 31 de agosto de 2021, de http://mastor.cl/blog/wp-content/ uploads/2018/02/Andreu.-analisis-decontenido.-34-pags-pdf.pdf

Boni, A., Belda-Miquel, S., \& Pellicer-Sifres, V. (2018). Innovación transformadora. Propuestas desde la innovación social colectiva para el desarrollo humano. RECERCA. Revista De Pensament I Anàlisi, (23), 67-94. https://doi. org/10.6035/Recerca.2018.23.4

Buckland, H., y Murillo, D. (2014). La Innovación Social en América Latina. Marco conceptual y agentes. Fomin.

Canter, L. (1997). Manual de evaluación de impacto ambiental: técnicas para la elaboración de estudios de impacto. McGraw-Hill.

Cohen, E., y Martínez, R. (2002). Formulación, evaluación y monitoreo de proyectos sociales. División de desarrollo social. CEPAL
Echeverría, J. (2008). El Manual de Oslo y la innovación social. ARBOR Ciencia, pensamiento y cultura, 610-618.

Fernández, V. (1993). Guia metodológica para la evaluación de impacto ambiental (segunda ed.) Madrid: Mundi-prensa.

Franks, D. (2012). Evaluación del impacto social de los proyectos de recursos. Queensland.

Franks, D. (2011). Management of the Social Impacts of Mining. Littleton, Colorado: SME Mining Engineering Handbook.

Garmendia, Salvador A.; Salvador, A.; Crespo, C., y Garmendia, L. (2005). Evaluación de impacto ambiental. Madrid: Pearson - Prentice Hall.

Hernández, R. (2014). Metodología de la investigación. Madrid: McGraw Hill.

Holsti, O. (1969). Content analysis for the social sciences and humanities. Addison-Wesley Pub. Co.

Mulgan, G., y Sanders, B. (2007). Social Innovation: What it is, why is matters and how it can be accelerated.

Navarro, H. (2005). Manual para la evaluación de impactos de proyectos sociales y programas de lucha contra la pobreza. Santiago, Chile.

Philis, J.; Deiglmeier, K., \& Miller, D. (2008). Rediscovering Social Innovation. Stanford Soial Innovation Review.

Prandi, M. y Cano, J. (2015). Coinnovación social. Claves para innovar desde la colaboración.

Proyecto Nasa (16 de 01 de 2019). Proyecto Nasa. R e c u p e r a d o de http://www.proyectonasa. org/index.php? option =com tent\&view=article \&id=35\&ltemid $=212$

Proyecto Nasa (2019a). Proyecto Nasa. Obtenido de Proyecto

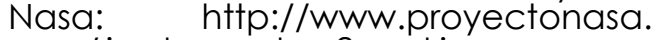
org/index.php? option=com tent\&view $=$ article \&id $=50 \&$ ltemid $=197^{-}$

Quijano, G.; Ibarvo, V.; Sánchez Basualdo, R., y Jiménez, G. (2017). La Innovación Social como estrategia para el desarrollo de comunidades rurales con población indígena en los estados de Sonora y Chihuahua, México. Revista de investigación académica sin frontera. (26) ISSN: 2007-8870. 
Sánchez,
social y solidaria en el en enpacio eurolatinoamericano. Innovación social para la internacionalización de cooperativas. Cooperativismo y desarrollo.

Schutt, R. (2001). Investigating the social world. The process and practice of research.

Stufflebeam, D. (1999). Foundational Models for 21 st century program evaluation. Michigan, USA.
Universidad Católiça de Chile (2012). La innovación social en Chile y el rol del estado en su desarrollo. Santiago.

Vanclay, F.; Esteves, A.; Aucamp, I., y Franks, D. (2015). Evaluación de Impacto Social: Lineamientos para la evaluación y gestión de impactos sociales de proyectos. Queensland

Westley, F. (2009). Social Innovation: an agency based approach to managing for resilience.

\section{(c) $(1) \Theta \ominus$

